\title{
CONSTRUÇÃO DE TECNOLOGIA EDUCATIVA EM AMBULATÓRIO DE RADIOTERAPIA
}

VASCONCELOS, C.B.H. ${ }^{1}$; BARBOSA, J.E.C. ${ }^{2}$; FREITAS, F.M.C. ${ }^{3}$; MAGALHÃES, T.L. ${ }^{4}{ }^{\&}$ RODRIGUES, A.B. ${ }^{5}$

${ }^{1}$ Enfermeira graduada pela Universidade Federal do Ceará (UFC), residente em Cancerologia pelo Instituto do Câncer do Ceará (ICC). Email: cecilia.bhv@gmail.com; ${ }^{2}$ Acadêmica de enfermagem pela Universidade Federal do Ceará (UFC), monitora da disciplina de

Laboratório de Fundamentos de Enfermagem. E-mail: jessycaelaine53@gmail.com; ${ }^{3}$ Enfermeira graduada pela Universidade Federal do Ceará (UFC). E-mail: nandamcfreitas60@gmail.com; ${ }^{4}$ Enfermeira graduada pela Universidade Federal do Ceará (UFC), residente em Cancerologia pelo Instituto do Câncer do Ceará (ICC). E-mail: tayannelmagalhaes@ gmail.com; ${ }^{5}$ Enfermeira, Doutora em enfermagem pela

Universidade Federal de São Paulo (USP), Especialista em enfermagem oncológica, Professora do curso de graduação em enfermagem de Universidade do Ceará, Coordenadora do projeto de extensão Liga Acadêmica de Oncologia. E-mail: andreabrodrigues@ gmail.com

Artigo submetido em 07/10/2017

\section{RESUMO}

O objetivo deste estudo foi relatar a experiência da construção de uma tecnologia educativa direcionada a orientação de pacientes oncológicos sob tratamento radioterápico. Trata-se de relato de experiência, desenvolvida em uma unidade de alta complexidade em oncologia (UNACON) do Ceará, com coleta de dados realizada em quatro etapas: primeira oficina com as enfermeiras do serviço, revisão integrativa da literatura para identificação dos efeitos colaterais da radioterapia e intervenções de enfermagem, segunda oficina com as enfermeiras para elaboração final da tecnologia educativa construída coletivamente. $\mathrm{O}$ período da pesquisa compreendeu de fevereiro a abril de 2016. O arcabouço teórico para construção do instrumento foi baseado na teoria de Doak, Doak, Rook. Foi construída tecnologia educativa que contemplou o que é câncer, o que é radioterapia, finalidades da radioterapia, e os efeitos colaterais gerais mais incidentes que acometem os pacientes sob tratamento radioterápico: radiodermatite, fadiga e inapetência. A tecnologia compõe-se por sete partes subdivididas nos seguintes tópicos: $\mathrm{O}$ que é Câncer? O que é radioterapia? Quais são os benefícios da radioterapia? Como é feita a radioterapia externa ou teleterapia? Recomendações importantes; Efeitos colaterais da radioterapia e Contatos Institucionais.

PALAVRAS-CHAVE: Radioterapia. Pesquisa nos serviços de saúde. Enfermagem oncológica. Pacotes de assistência ao paciente. Assistência ambulatorial.

\section{CONSTRUCTION OF EDUCATIONAL TECHNOLOGY IN RADIOTHERAPY AMBULATORY}

\begin{abstract}
Study reported the experience of building educational technology directed to the orientation of cancer patients under radiotherapy treatment. Experience report, developed in a UNACON of Ceará. Data collection performed in two stages: first integrative literature review to identify side effects of radiotherapy and nursing interventions, second workshop with nurse for final elaboration of educational technology. The research period ranged from February to April 2016. The theoretical framework for the construction of the instrument was
\end{abstract}

based on the theory of Doak, Doak, Root. Educational technology was built covering cancer and radiotherapy, radiotherapy purposes and more incident side effects in patients undergoing radiotherapy. Technology consists of seven parts subdivided into the following topics: What is Cancer? What is radiotherapy? What are the benefits of radiotherapy? How is external radiotherapy or teletherapy performed? Important recommendations; Side effects of radiotherapy and Institutional Contacts.

KEYWORDS: Radiotherapy. Research in health services. Oncological nursing. Patient care packages. Ambulatory care. 


\section{INTRODUÇÃO}

Para o tratamento dos tumores malignos, a radioterapia é uma modalidade terapêutica bastante utilizada, sendo a dose determinada de acordo com a extensão do tumor e do tipo de câncer diagnosticado, o que influencia também na quantidade de sessões realizadas. (INCA,2017). É um tratamento que utiliza radiação ionizante, produzida por aparelhos ou emitida por radioisótopos com a finalidade de promover o controle ou erradicação da doença.

No entanto, efeitos colaterais a esse tratamento podem ocorrer como a radiodermatite, a fadiga e a inapetência (SCHNEIDER et, al 2013). Em aproximadamente 80\% dos casos, a radiação induz à radiodermatite, uma inflamação da pele que causa dor, provocando, em alguns casos, a interrupção do tratamento (LENHANI et, al, 2014). Outros efeitos são, ainda, decorrentes da aplicação da radiação de acordo com a área anatômica irradiada. Na região de cabeça e pescoço, por exemplo, podem ocorrer disfagia, odinofagia, disgeusia, xerostomia e mucosite (RODRIGUES; MIYAHIRA; 2015).

Os efeitos colaterais apresentados pelo paciente em tratamento radioterápico devem ser acompanhados por meio de uma rotina sistemática de avaliações diárias pela enfermeira e pelo médico durante as consultas semanais (LENHANI et, al, 2014)

A consulta de enfermagem, sendo atividade privativa do enfermeiro, utiliza componentes do método científico para identificar situações de saúde/doença, prescrever e implementar medidas de enfermagem que contribuam para a prevenção, promoção e recuperação da saúde do indivíduo, família e comunidade. Em todos os níveis de assistência à saúde, seja em instituição pública ou privada, a consulta de enfermagem deve ser obrigatoriamente desenvolvida, conforme a resolução no 358/2009 do Conselho Federal de Enfermagem (BRASIL, 2009).

Ao realizar a consulta de enfermagem, o enfermeiro deve utilizar-se de materiais educativos como cartilhas, folhetos e manuais de cuidados, que facilitam a compreensão por parte do cliente.

O enfermeiro tem na sua natureza ser um educador, ao sistematizar e individualizar o cuidado e voltar-se não somente para a doença, mas também influenciar sobre o estilo de vida das pessoas, tornando-as sujeitos de suas próprias decisões. A enfermagem tem sido importante ao procurar novas metodologias a fim de alcançar melhorias na qualidade de vida e da assistência ao paciente, mediante atividades educativas de saúde e intervenções apropriadas (LOPES; ANJOS; PINHEIRO, 2009).

A educação em saúde é definida como um processo educativo de construção de 
conhecimentos em saúde o qual tem em mente à apropriação temática pela população e não à profissionalização ou à carreira na saúde; e é tida também como um conjunto de práticas do setor que busca contribuir aumentando a autonomia das pessoas no autocuidado e no debate com os profissionais e os gestores a fim de obter uma atenção de saúde de acordo com suas necessidades (BRASIL, 2009).

O enfermeiro ou outro profissional de saúde, que lida com educação em saúde têm como atribuição escolher, selecionar e preparar a mensagem e determinar a melhor forma de transmissão da comunicação, desde que possibilite a uma comunicação efetiva e garanta uma assistência que atenda às necessidades do paciente. Assim, um material, se for bem escrito, ou uma informação de fácil compreensão melhora o conhecimento e a satisfação do paciente, desenvolve suas atitudes e habilidades, facilita na autonomia, promove maior adesão, ajuda-os a entender como as próprias ações refletem no seu padrão de saúde, favorece sua tomada de decisão, ou seja, é uma forma de promover saúde (MOREIRA; NÓBREGA; SILVA, 2003).

Diante desse contexto e mediante a inserção dos acadêmicos de enfermagem que compõem o projeto de extensão Liga Acadêmica de Oncologia pertencente à Universidade Federal do Ceará do país em uma UNACON foi levantada a necessidade de construir uma tecnologia educativa para auxiliar no processo de educação em saúde aos pacientes durante a consulta de enfermagem em ambulatório de radioterapia. Sendo assim, o objetivo deste estudo foi elaborar uma tecnologia educativa para auxiliar no processo de educação em saúde durante consulta de enfermagem a pacientes em tratamento radioterápico ambulatorial em uma UNACON.

\section{MÉTODO}

Trata-se de relato de experiência de construção de uma tecnologia educativa que foi o resultado final de um trabalho que envolveu um conjunto de ações abstratas ou concretas, não podendo ser considerada apenas como algo concreto, como um produto palpável, pois permeou todo o processo de trabalho em saúde, contribuindo na construção do saber, se apresentando desde o momento da ideia inicial, da elaboração e da implementação do conhecimento, como também, do resultado dessa mesma construção. A tecnologia é considerada ao mesmo tempo processo e produto (ROCHA et al, 2008).

O contexto do estudo se deu em um ambulatório de radioterapia de uma UNACON, na região Nordeste do Brasil, onde são atendidos aproximadamente 300 pacientes por dia, os quais, em sua maioria, são portadores de cânceres de próstata, mama, cólon e reto e cabeça e pescoço. 
A equipe de saúde é composta por médicos, enfermeiros, técnicos de enfermagem, nutricionista e psicólogo, assistente social, fonoaudiólogo, terapeuta ocupacional e fisioterapeuta.

Foram percorridas as etapas de concepção, organização de uma oficina com as enfermeiras do serviço, revisão integrativa da literatura, construção da tecnologia a partir das informações identificadas na revisão integrativa de literatura, segunda oficina com as enfermeiras para verificação das informações científicas relevantes e aprovação pelo marketing institucional da UNACON.

A fase de concepção ocorreu em fevereiro de 2016, onde foi definido o problema de pesquisa em uma reunião com a enfermeira coordenadora da unidade de radioterapia, onde pactuou-se a elaboração, em conjunto, de uma tecnologia educativa para auxiliar o processo de educação em saúde durante as consultas de enfermagem que pudessem atender às necessidades dos pacientes atendidos no serviço. Foi paralelamente realizada uma oficina em sala privativa com as enfermeiras do ambulatório para conhecer os recursos materiais, humanos, características do serviço e da clientela atendida.

O marco teórico para orientação da construção da tecnologia educativa foi embasado na teoria da educação de Doak, Doak, Root (1996). Considerando-se que a informação escrita é destinada a informar ou capacitar, a linguagem e os recursos visuais precisam ser claros e simples, e o texto bem organizado. Dessa forma, teremos as instruções de saúde mais bem compreendidas por muitos (DOAK; DOAK; ROOT, 1996).

Algumas orientações são imprescindíveis para os passos de planejamento de construção dos materiais: 1) Definir o público-alvo e, apesar de parecer ser uma etapa óbvia, muitas vezes, acaba por ser negligenciada; 2) Limitar e definir os objetivos de aprendizagem, possivelmente umas das etapas mais difíceis do processo. Os objetivos devem ser compatíveis exatamente com as ações e os comportamentos que se deseja obter com a intervenção educacional. Eles podem ser definidos primeiramente fazendo-se uma avaliação das necessidades; 3) Usar palavras comuns ao vocabulário evitando termos técnicos que dificultam a compreensão, e caso utilize essas palavras é necessário exemplificar para que todos possam entender. Bem como escrever sentenças curtas por serem mais fáceis de ler e compreender; 4) Incluir interações que possam tornar as orientações mais simples de aprender e lembrar, contudo é uma das características menos utilizada, e também as revisões; 5) Por fim, testar para assegurar uma garantia de qualidade (DOAK; DOAK; ROOT, 1996).

$\mathrm{Na}$ fase de revisão integrativa da literatura teve-se como intuito responder à seguinte questão norteadora: "Quais os efeitos colaterais que acometem pacientes em tratamento 
radioterápico e as intervenções de enfermagem com bom nível de evidência científica para manejo dos mesmos?".

A busca foi feita nas bases de dados Literatura Latino-Americana e do Caribe em Ciências da Saúde (LILACS), Medical Literature Analysis and Retrieval System Online (MEDLINE), Cumulative Index to Nursing and Allied Health Literature (CINAHL), Scientific Eletronic Library Online (SciELO). Ademais, também foram utilizados sites científicos de associações específicas da especialidade Oncologia, a saber: Instituto Nacional do Câncer (INCA), Radiation Therapy Oncology Group (RTOG), American Society of Clinical Oncology (ASCO), Oncology Nurses Society (ONS) e National Cancer Institute (NCI). Foram utilizadas publicações no período de 2011 a 2016, nos idiomas português, inglês e espanhol com os descritores indexados nos Descritores em Ciências da Saúde (DeCS) da Biblioteca Virtual em Saúde e do Medical Subject Headings (MeSH) da National Library of Medicine: Oncologia, Câncer, Radioterapia. Nos sites das associações, a busca foi guiada pelas palavras chave "Efeitos Colaterais", "Radioterapia" e "Intervenções" nos mesmos idiomas supracitados. Excluiu-se editoriais, revisões de literatura e artigos não disponíveis na íntegra. Também foram pesquisados livros texto, a saber: Radioterapia em oncologia (editora Atheneu), Oncologia multiprofissional: bases para assistência (editora Manole) e o livro Nursing Interventions Classification (NIC) (editora Elsevier), que representa padrão de prática internacional para intervenções no âmbito da enfermagem.

A partir dos efeitos colaterais e intervenções de enfermagem identificados na revisão foi organizada uma segunda oficina com as pesquisadoras e as enfermeiras em sala privativa na própria UNACON, a qual teve como objetivos discutir sobre os itens relevantes para constar na tecnologia educativa, indicando todas as adequações para torná-lo um modelo de fácil compreensão e implementação que favorecesse o cuidado profissional exercido pelo enfermeiro.

$\mathrm{O}$ instrumento também foi submetido à avaliação pelo marketing institucional da própria UNACON, tendo recebido tratamento iconográfico atendendo ao padrão da própria instituição.

\section{RESULTADOS}

Da análise das informações dos pacientes coletadas durante a primeira oficina com as enfermeiras pode-se evidenciar que o perfil sóciodemográfico da população atendida no serviço é de pessoas com ensino fundamental incompleto, trabalhadores, provenientes de outras cidades 
do estado do Ceará. As topografias anatômicas mais incidentes de câncer que são tratadas no ambulatório de radioterapia foram próstata, cabeça e pescoço e mama.

Com o objetivo de levantamento de dados relevantes para constar na tecnologia educativa, identificou-se por meio da revisão integrativa um total de 408 publicações, sendo excluídas 384 por não atenderem aos critérios de inclusão. Do total de publicações incluídas $(\mathrm{n}=24)$, a maioria foi identificada no base CINAHL $(5 ; 20,83 \%)$ e na base LILACS $(1 ; 4,16 \%)$ As outras publicações $(18 ; 75 \%)$ foram identificadas nos sites das associações ASCO e ONS e nos livros-texto.Os efeitos colaterais/complicações identificados foram categorizados a seguir.

Quadro 1 - Efeitos colaterais/complicações decorrentes da radioterapia identificados na revisão integrativa. Fortaleza, Ceará, 2016.

\begin{tabular}{|c|c|}
\hline $\begin{array}{c}\text { Efeitos colaterais Gerais independentes da área de } \\
\text { tratamento }\end{array}$ & $\begin{array}{c}\text { Efeitos colaterais específicos de acordo com a área } \\
\text { de tratamento }\end{array}$ \\
\hline Radiodermatite, fadiga, inapetência. & Mucosite oral, xerostomia e náusa. \\
\hline
\end{tabular}

A construção do folder iniciou-se pela definição do que é câncer, visto que a maior parte da população não tem conhecimento de como se desenvolve a neoplasia.

Figura 1 - O que é Câncer

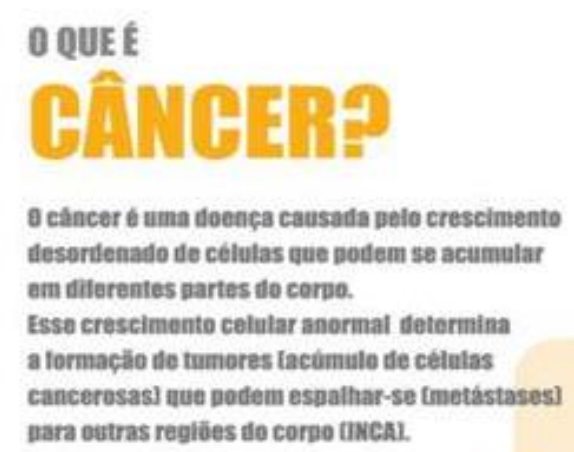

Logo após, seguiu-se com considerações sobre o que é radioterapia e como é feita a radioterapia. Deve-se levar em consideração que essa modalidade de tratamento difere das demais modalidades de tratamento na área da saúde por diversos motivos. Um deles é que é aplicado de forma fracionada, em geral, cinco dias por semana demandando do paciente idas diárias até o serviço de saúde e utiliza um aparelho semelhante a um aparelho de raio X para emissão da radiação (RODRIGUES, 2015). Sendo assim, considera-se a importância de algumas orientações gerais, como utilização de produtos tópicos (HERR, 2013), visto que a não adesão compromete a eficácia do tratamento. 
Figura 2 - O que é Radioterapia?

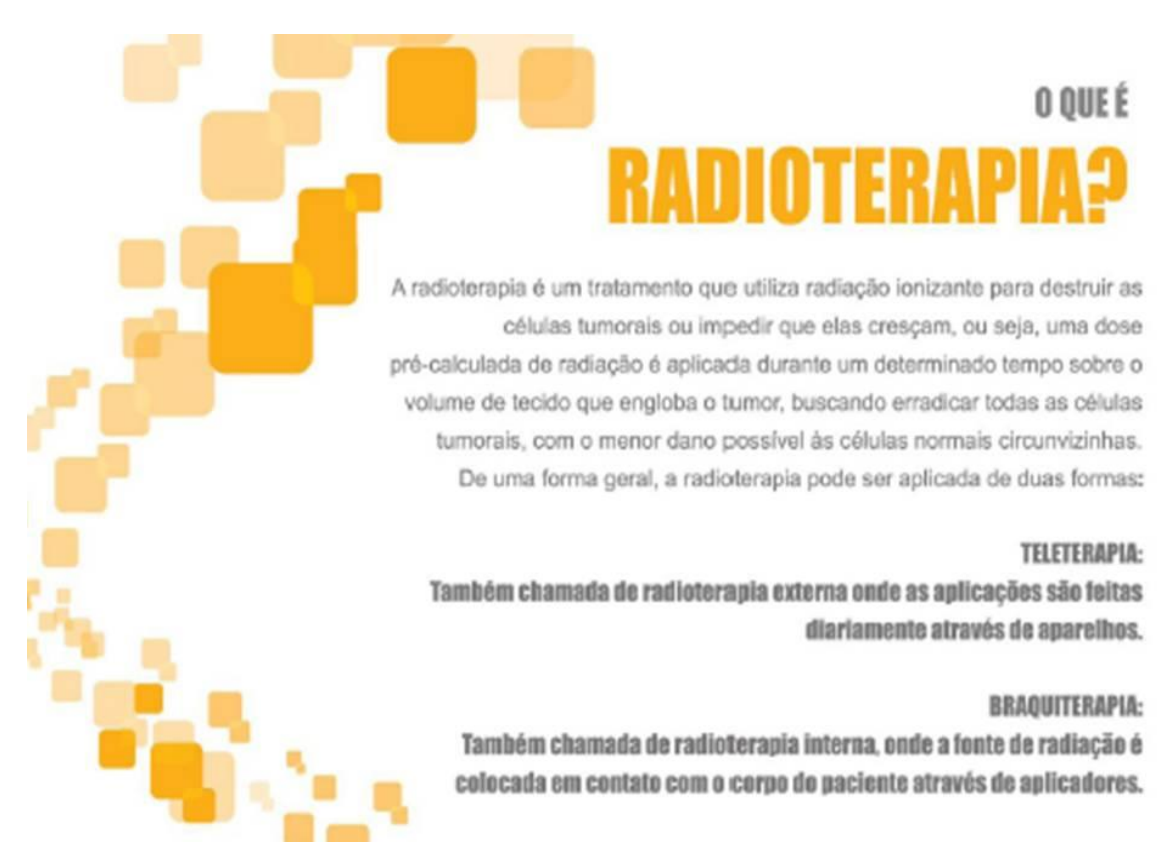

Figura 3 - Como é feita a teleterapia?

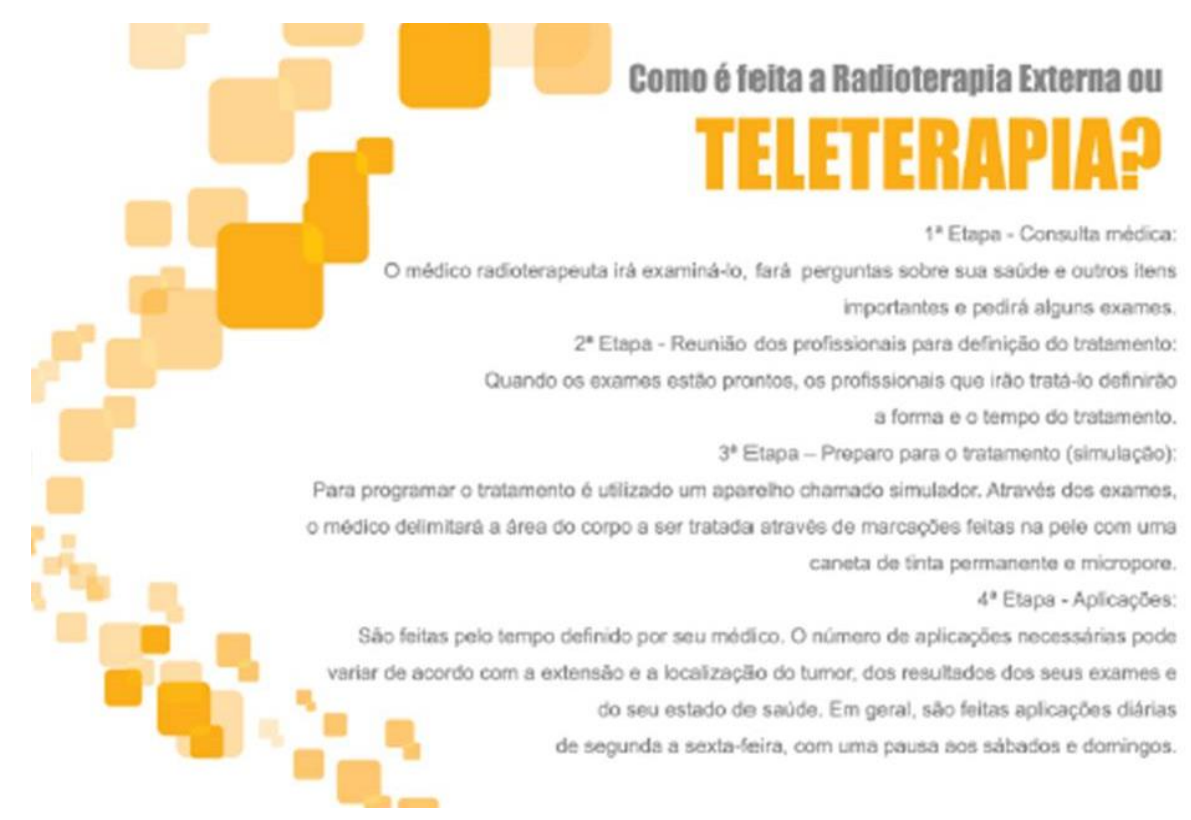

Logo após, inicia-se orientações com nível de evidência científica I ou II para prevenção e manejo dos efeitos colaterais mais comuns identificados na literatura científica, a saber: radiodermatite, fadiga e inapetência.

As intervenções de enfermagem foram selecionadas a partir das publicações encontradas nas bases de dados selecionadas, livros-texto e no livro da NIC.

Pode-se visualizar na Figura 3 o trecho da tecnologia educativa que aborda as intervenções para evitar/manejar a radiodermatite. 
Figura 4 - Manejo da Radioterapia

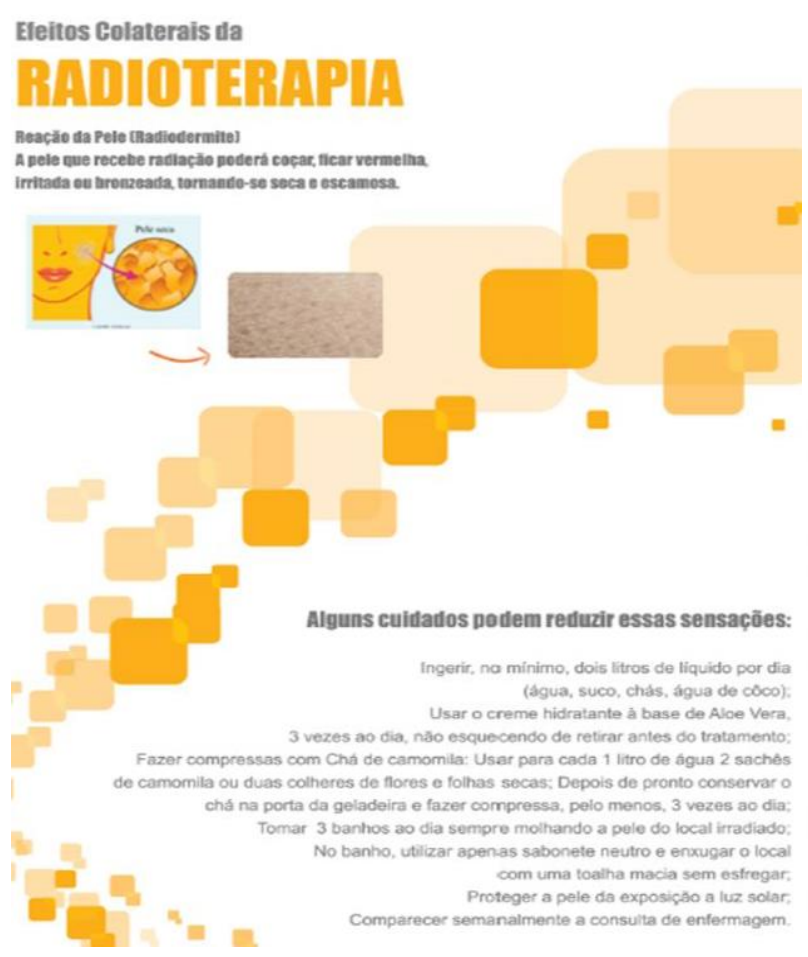

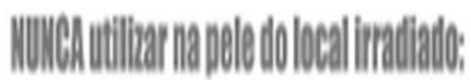

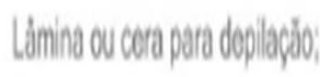

Cremes, alco, descolorante, pertume ou qualquer outa

substanca que contentha alcoo:

Esparadrapo ou qualuere tipo de adesivo:

Bolsa de dgua quente ou de geglo:

Näo trequentar saunas:

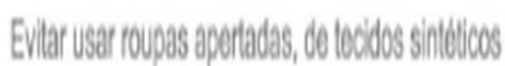

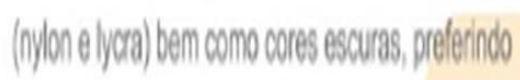

sempre rovpas leves 8 contortituis.

Como limitações do estudo pode-se enumerar a dificuldade de obtenção de pesquisas relacionadas à assistência de enfermagem em radioterapia para discussão dos dados. Ponderase também que esse folder atende às necessidades de pacientes sob tratamento radioterápico em geral. Sendo assim, pretende-se futuramente avançar com a construção de outras tecnologias educativas para efeitos colaterais específicos de cada área de tratamento (cabeça e pescoço, tórax, abdome e pelve) para atender outra demanda de pacientes do serviço.

\section{CONCLUSÃO}

A partir da revisão integrativa e das oficinas realizadas com as enfermeiras do serviço, foi possível construir uma tecnologia educativa para auxiliar na educação em saúde em contexto ambulatorial de radioterapia. O folder construído é composto por sete partes subdivididas nos seguintes tópicos: O que é Câncer? O que é radioterapia? Quais são os benefícios da radioterapia? Como é feita a radioterapia externa ou teleterapia? Recomendações importantes; Efeitos colaterais da radioterapia e Contatos Institucionais.

Acredita-se que dessa forma, as enfermeiras possam prestar uma assistência de enfermagem pautada no método sistematizado, além de poder proporcionar uma ferramenta educativa para minimizar agravos à saúde decorrente do tratamento radioterápico ambulatorial. 


\section{REFERÊNCIAS}

ANDRADE, M; CLAPIS, MJ; NASCIMENTO, TG; GOZZO, TO; ALMEIDA, AM.

Prevenção de reações de pele devido à teleterapia em mulheres com câncer de mama: revisão integrativa. Rev. Latino-Am. Enfermagem maio-jun. 2012;20(3):[8 telas]. Disponível em: http://www.scielo.br/pdf/rlae/v20n3/pt_a24v20n3.pdf. Acesso em 28 de abr 2017

ECHER, IC. Elaboração de manuais de orientação para o cuidado em saúde. Rev. LatinoAm. Enfermagem, Ribeirão Preto, v. 13, n. 5, p. 754-757, out. 2005. Disponível em http://www.scielo.br/scielo.php?script=sci_arttext\&pid=S0104-

$11692005000500022 \& \operatorname{lng}=$ pt\&nrm=iso. Acessos em 29 abr. 2017.

HERR, GE; KOLANKIEWICZ, ACB; BERLEZI, EM; GOMES, JS; MAGNAGO, TSBS; ROSANELLI, CP; LORO, MM. Avaliação de Conhecimentos acerca da Doença Oncológica e Práticas de Cuidado com a Saúde. Revista Brasileira de Cancerologia 2013; 59(1): 33-41. Disponível em: http://www.inca.gov.br/rbc/n_59/v01/pdf/06-avaliacao-de-conhecimentosacerca-da-doenca-oncologica-e-praticas-de-cuidado-com-a-saude.pdf. Acesso em 28 de abr. 2017.

INSTITUTO NACIONAL DO CÂNCER JOSÉ ALENCAR GOMES DA SILVA. Orientações para pacientes que se submetem a sessões de radioterapia. [Internet]. 2017. Disponível em: http://www.inca.gov.br/conteudo_view.asp?id=115. Acesso em 20 de abr. de 2016.

LENHANI, BE; GONZAGA, AKLL; PADILHA, RA; SILVA, AVS; BAY, EOS. Intervenções de enfermagem a paciente com radiodermite grau IV: relato de caso. Rev enferm UFPE on line[Internet] 2014 Jul; 8 (7): 2089-94. Disponível em: http://www.periodicos.ufpe.br/revistas/revistaenfermagem/article/view/9888. Acesso em 20 de out. 2016

ROCHA, PK; PRADO, ML; SILVA, DMGV. Pesquisa Convergente Assistencial: uso na elaboração de modelos de cuidado de Enfermagem. Revista Brasileira de Enfermagem [Internet]. 2012 Nov-Dez; 65 (6): 119-25. Disponível em: http://www.scielo.br/pdf/reben/v65n6/a19v65n6.pdf. Acesso em 28 de abr. 2016

RODRIGUES, AB; MIYAHIRA, RC. Bases da Radioterapia. In: RODRIGUES, AB; MARTIN, LGR; MORAES, MW. Oncologia multiprofissional: bases para assistência. São Paulo: Manole; 2015.

SCHNEIDER, F; PEDROLO, E; LIND, J; SCHWANKE, AA; DANSKI, MTR. Prevenção e tratamento de radiodermatite: uma revisão integrativa. Cogitare Enferm. [Internet]. 2013 Jul-Set; 18(3): 579-86. Disponível em:

http://revistas.ufpr.br/cogitare/article/viewFile/33575/21073. Acesso em 20 de nov. 2016. 